\title{
Neurosifilis meningovascular como causa de accidente cerebrovascular en paciente joven: reporte de caso
}

José Mauricio Ocampo-Chaparro* César Augusto Ríos-Quintero** Jorge Eliecer Álvarez-Payares*** Jenny Patricia Muñoz-Lombo****

\begin{abstract}
* Médico Familiar y Geriatra. Magíster en Epidemiología y Gerontología social. Departamento de Medicina Familiar. Universidad del Valle. Departamento de Medicina Interna. Grupo Interinstitucional de Medicina Interna (GIMI 1). Universidad Libre. Cali. Colombia. **Médico internista. Grupo Interinstitucional de Medicina Interna (GIMI 1). Universidad Libre. Cali. Colombia.

***Médico Internista Infectólogo. Departamento de Medicina Interna. Facultad de Salud. Universidad del Valle. Cali. Colombia. **** Médica internista, Magíster en Epidemiología. Grupo Interinstitucional de Medicina Interna (GIMI 1). Universidad Libre. Cali. Colombia. Correspondencia: José Mauricio Ocampo Chaparro. Dirección: Carrera 72 \#13A-56. Conjunto residencial Ponte Vedra, apartamento 501 A. Cali. Colombia. Teléfono: (+57) 313750 0816. Correo electrónico: jose.m.ocampo@correounivalle.edu.co.
\end{abstract}

Resumen

El accidente cerebrovascular en adultos jóvenes es un evento raro presente en menos del $5 \%$ de los casos a nivel mundial, representando un reto diagnóstico debido a las múltiples etiologías posibles, entre ellas las infecciones. Del $15 \%$ al $40 \%$ de los pacientes con sífilis no tratada pueden desarrollar sífilis terciaria con manifestaciones principalmente neurodestructivas, descritas en la sífilis tardía pero con meningitis crónica y accidente cerebrovascular de tipo meningovascular en su fase temprana. Se presenta el caso de un paciente masculino de 40 años con antecedente de cambios de comportamiento, alucinaciones auditivas e insomnio, quien consulta a urgencias por presentar hemiplejia derecha, afasia motora, lesiones palmo-plantares y roseta en glande asociada a adenopatías inguinales. Dados los hallazgos imagenológicos y de líquido cefalorraquídeo se estableció diagnóstico de accidente cerebrovascular isquémico de circulación cerebral anterior izquierda secundario a neurosífilis meningovascular, con confirmación de inmunosupresión por virus de inmunodeficiencia humana. MÉD.UIS.2019;32(2):53-8

Palabras clave: Treponema pallidum. Neurosífilis. Accidente cerebrovascular. Líquido cefalorraquídeo. Síndrome de Inmunodeficiencia Adquirida. Penicilina.

\section{Meningovascular neurosyphilis as a cause of stroke in a young patient: case report}

\section{Abstract}

Stroke in young adults is a rare event present in less than $5 \%$ of cases worldwide, representing a diagnostic challenge due to the multiple possible etiologies, including infections. $15 \%$ to $40 \%$ of patients with untreated syphilis may develop tertiary syphilis; with mainly neurodestructive manifestations, described in late syphilis, but in its early stage with chronic meningitis and meningovascular stroke. The case of a 40-year-old male patient with a history of behavior changes, auditory hallucinations and insomnia is presented, who consults the emergency department for presenting right hemiplegia, motor aphasia, palmoplantar lesions and rosette in glans associated with inguinal adenopathies. Given the imaging and cerebrospinal fluid findings, a diagnosis of ischemic stroke of the left anterior cerebral circulation was established, secondary to meningovascular neurosyphilis, with confirmation of immunosuppression due to human immunodeficiency virus. MÉD.UIS.2019;32(2):53-8

Keyword: Treponema pallidum. Neurosyphilis. Stroke. Cerebrospinal fluid, Acquired Immunodeficiency syndrome. Penicillin.

Artículo recibido el 20 de noviembre del 2017 y aceptado para publicación el 09 de diciembre del 2018 
¿Cómo citar este artículo?: Ocampo-Chaparro JM, Ríos-Quintero CA, Álvarez-Payares JE, MuñozLombo JP. Neurosífilis meningovascular como causa de accidente cerebrovascular en paciente joven: reporte de caso. MÉD.UIS.2019;32(2):53-8. doi: 10.18273/revmed.v32n2-2019007

\section{Introducción}

El Accidente Cerebrovascular (ACV) en adultos jóvenes es un evento raro, definido como una lesión cerebral resultado de un agravio vascular que, para el caso isquémico, es resultado de un flujo sanguíneo insuficiente que conlleva a daño neuronal y muerte celular. Se presenta entre los 18 a 50 años; sin embargo, hay dificultad en la operacionalización de esta definición, encontrando variedad en los puntos de corte en la literatura, que pueden llegar hacer desde los 15 hasta los 45 años $^{1-2}$.

Uno de cada diez casos de ACV isquémicos reportados a nivel mundial ocurre en adultos jóvenes, con una incidencia de más de dos millones de casos anuales ${ }^{1}$ con variaciones según los intervalos de edad, siendo baja en menores de 35 años ( 1,2 a 4,5 casos por cada 100.000 habitantes), pero directamente proporcional al incremento de la edad con incidencias hasta del 32,9 por cada 100.000 habitantes entre los 45 y 49 años de edad ${ }^{3}$.

En Latinoamérica, el $12 \%$ de las enfermedades cerebrovasculares ocurren en menores de 45 años $^{4}$. En Colombia, para el 2014, la incidencia de ACV isquémico fue de 22,45 casos por cada 100000 habitantes en el grupo etario de los 35 a 39 años, datos equiparables a los mayores de 50 años. Para los hombres, los casos se presentaron desde los 15 años con un pico, al igual que en las mujeres, de 33,57 casos por 100.000 habitantes en los pacientes entre 35 a 39 años. En cuanto a la mortalidad, en ambos sexos este era directamente proporcional al incremento de la edad5. A pesar de lo anterior, la mayoría de los casos tiene un adecuado pronóstico, pero con importantes impactos a nivel socioeconómico dado por la discapacidad secundaria por la pérdida de años productivos por la alta expectativa de vida de estos pacientes'․

Las causas de la patología en esta población son heterogéneas, independiente a los factores de riesgo clásicos cardiovasculares como el tabaquismo, la hipertensión, la diabetes mellitus y la hiperlipidemia ${ }^{6}$. Se deben considerar causas ateroescleróticas, cardioembólicas, pero también genéticas, autoinmunes, hematológicas, oncológicas e infecciosas'. De este último se han identificado múltiples agentes causales tales como los virus de inmunodeficiencia humana (VIH) y herpes zoster, infecciones micóticas como la criptococosis y la aspergilosis, y bacterianas principalmente por tuberculosis y sifilis².

La sífilis, conocida desde tiempo inmemorables como la gran imitadora, es una enfermedad infectocontagiosa causada por la espiroqueta Treponema pallidum subsp. pallidum, la cual se transmite por vía sexual y vertical cursando con varias etapas clínicas: la sífilis temprana, en donde la infección es transmitida sexualmente e incluye la sífilis primaria; secundaria y latente temprana. Y la etapa latente tardía y la sífilis terciaria siendo esta última menos común debido al amplio uso de antibioticoterapia?.

Aproximadamente 17,7 millones de personas en el mundo entre las edades de 15 a 49 años tenían sífilis para el 2012, con una incidencia anual de alrededor de 5,6 millones de casos afectando en gran medida países de África y América, donde las incidencias para 2012 estaban alrededor del 1843000 y 937700 respectivamente ${ }^{8}$.

Con relación a la neurosífilis, las manifestaciones clínicas son amplias y heterogéneas, encontrando entre ellas síntomas oftalmológicos o auditivos, parálisis de pares craneales, disfunción cognitiva, deficiencias motoras o sensoriales, signos de meningitis o de accidente cerebrovascular ${ }^{9}$. Se reporta una mayor predisposición a su desarrollo en pacientes con coinfección con $\mathrm{VIH}^{10}$, donde no solo ocurre durante las manifestaciones tardías sino también durante las tempranas dado a que la penetración de la barrera hematoencefálica por la espiroqueta se genera hasta en el $40 \%$ de los casos de sífilis sin tratamiento"1. Se debe tener alta sospecha diagnóstica en los usuarios de drogas intravenosas $y$ en hombres que tienen sexo con hombres siendo estos el $83 \%$ de todos los casos; por lo tanto, se observa una incidencia con crecimiento exponencial en este tipo de grupos poblacionales ${ }^{12,13}$. 
Gracias al diagnóstico precoz ante la necesidad de buscar activamente la enfermedad en las fases tempranas, junto con el tratamiento efectivo a base de penicilina, la incidencia de neurosífilis ha disminuido ostensiblemente ${ }^{14,15}$. La neurosífilis se desarrolla en un porcentaje cercano al $10 \%$ de los pacientes con sífilis temprana sin tratamiento ${ }^{16}$. De los pacientes $\mathrm{VIH}$ positivos, cerca del $15 \%$ tienen serología reactiva para sífilis y cerca del $2 \%$ padecen de neurosífilis ${ }^{17}$.

A pesar de esto, en Colombia se ha observado un incremento en la incidencia de la neurosífilis en las últimas décadas, pero se carecen de datos estadísticos aproximados por falta de un sistema de información adecuado, como sí se disponen en países desarrollados ${ }^{18}$. Más allá de estos datos estadísticos, solo hay reportes de casos y series de casos informados en nuestro país ${ }^{12,19}$ y las aproximaciones estadísticas las relacionan con la ineficiencia en las campañas de salud y comportamientos de riesgo, así como tratamientos insuficientes. En cuanto a la neurosífilis en pacientes con VIH, dada su baja incidencia, sumado a las limitaciones en infraestructura informática, no se cuentan con registros que relacionen las dos enfermedades en nuestro país, y en la literatura reportada en Colombia no se documenta la presencia de VIH en los casos publicados $^{20,21}$.

El objetivo de esta artículo es describir el comportamiento de la neurosífilis meningovascular como entidad causante de evento cerebrovascular en paciente joven, desde lo clínico y paraclínico, ya que este diagnóstico representa un reto clínico teniendo en cuenta que la sífilis es una entidad olvidada que logra pasar inadvertida.

\section{Presentación del caso}

Se trata de un paciente masculino de 40 años procedente de la ciudad de Cali, que refiere como antecedente personal relaciones sexuales con hombres y sin protección. Desde hacía tres meses manifestaba la presencia de alucinaciones auditivas complejas, insomnio y disminución de la agudeza auditiva, además de conducta negativa con aislamiento social, manifestado por familiares. Al mes de iniciados los síntomas presentó desviación de la comisura labial, disartria y marcha atáxica, alteraciones que se auto limitaron, razón por la cual no consultó. A los dos meses del evento descrito el paciente presenta crisis convulsiva tónico-clónico generalizada, sin afectación de movimientos oculares ni relajación de esfínteres, por lo que es llevado al servicio de urgencias de una clínica de tercer nivel en la ciudad de Cali. Al ingreso se encuentra hipertenso (TA: 140/90 mm Hg) y taquicárdico (FC: 96 latidos por minuto), evidenciando al examen físico unas lesiones hiperqueratósicas, descamativas, no supurativas tanto en palmas como en plantas (Ver figura 1) más lesiones húmedas en forma de roseta a nivel de glande (Ver figura 2) asociado a adenopatías inguinales. En el examen neurológico se encontró afasia motora, desviación de la comisura labial, hemiplejía derecha, y reflejos tendinosos aumentados sin signos meníngeos.
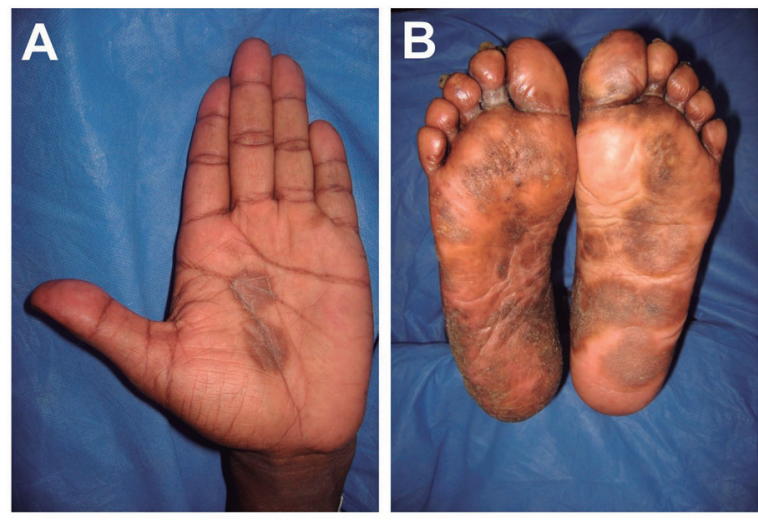

Figura 1. Lesiones tipo placas eritematosas, descamativas, hiperqueratósicas de bordes bien definidos, que comprometen palmas y plantas. Algunas de las lesiones tienen elementos en tiro al blanco. (A) en palmas, (B) en plantas

Fuente: autores

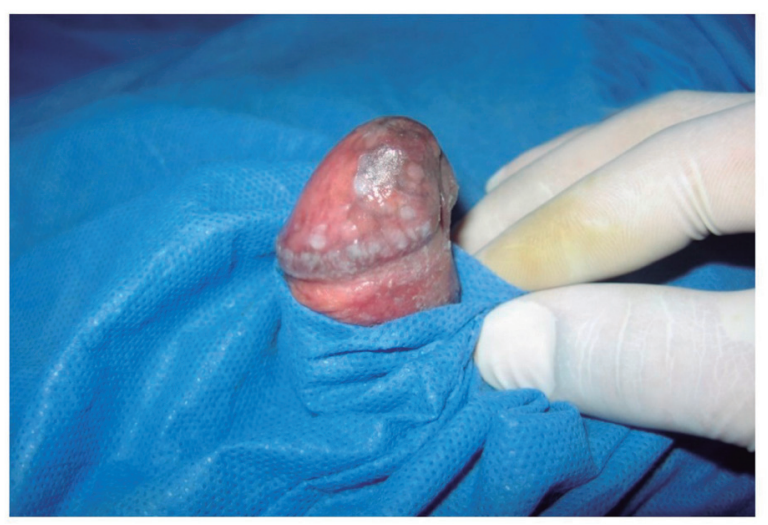

Figura 2. Lesiones tipo placa húmedas confluentes, distribuidas en el surco balano prepucial y en superficie del glande, de aspecto blanquecino, compatibles con condilomas planos, sugestivos de sífilis avanzada

Fuente: autores

Se consideró que el paciente cursaba con un síndrome convulsivo secundario a evento cerebrovascular, razón por la cual se inició manejo con fenitoína intravenosa a dosis de $15 \mathrm{mg} / \mathrm{kg}$ continuando con una 
dosis de mantenimiento de $250 \mathrm{mg}$ cada 8 horas con realización de tomografía axial computada cerebral simple y contrastada que reveló una hipodensidad fronto-temporal izquierda compatible con evento cerebrovascular isquémico (Ver figura 3).

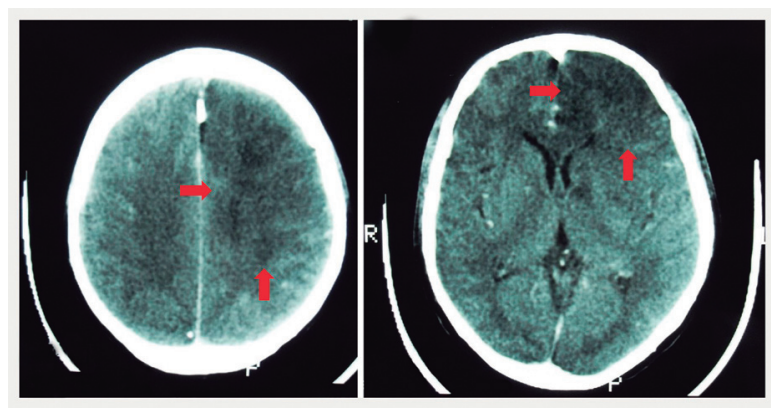

Figura 3. Tomografía axial computada (TAC) de cerebro simple y contrastada que evidencia hipodensidad en la región frontotemporal izquierda, con borramiento de los surcos y obliteración del ventrículo ipsilateral, con características de evento cerebral isquémico (flechas rojas).

Fuente: autores

Confirmada la sospecha diagnóstica se procedió a la búsqueda de la etiología; considerando su edad y los hallazgos al examen físico se solicitó serología RPR para sífilis en suero la cual fue reactiva 1:64 diluciones, con prueba treponémica tipo FTAABS positivo. Por este hallazgo se realizó punción lumbar que documentó líquido cefalorraquídeo (LCR) con hipoglucorraquia e hiperproteinorraquia con serología VDRL para sífilis en reactiva 1:8 diluciones. Con lo anterior, se consideró diagnóstico de neurosífilis meningovascular y se inició manejo con penicilina cristalina 4 millones de unidades endovenosa cada 4 horas por 14 días. Las lesiones cutáneas fueron manejadas por el servicio de dermatología con urea al 20\% y ácido salicílico tópicos por 14 días. Finalmente, se completó estudio con prueba presuntiva ELISA para detección de VIH tercera generación, que fue reportada como reactiva y confirmada con una segunda prueba de ELISA de cuarta generación, ratificando infección por VIH, con perfil inmunológico alterado por recuento de células CD4 de 250 células $/ \mathrm{mm}^{3}$ y carga viral plasmática (VIHRNA) de 10.000 copias/ml. Durante su hospitalización recibió consejería por psicología, terapia física y fonoaudiología.

Una vez se instauró tratamiento con penicilina se observó mejoría de los síntomas cognitivos a los 5 días, aunque persistió el déficit neurológico focal secundario al evento vascular, con hemiparesia derecha y discapacidad para la marcha. El paciente terminó el tratamiento para la neurosífilis de 14 días considerando la negativización en LCR del VDRL, por lo que egresó satisfactoriamente con posterior ingreso a un programa de atención integral para VIH/ sida para iniciar tratamiento.

\section{Discusión}

El ACV isquémico constituye una de las principales causas de discapacidad a nivel mundial y a pesar de la mejoría en la prevención primaria, diagnóstico oportuno y opciones de tratamiento, aún ocupa los primeros lugares en mortalidad ${ }^{19}$. Por su naturaleza y etiología heterogénea en personas adultas jóvenes, se hace necesario considerar un amplio arsenal de diagnósticos diferenciales resaltando las neuroinfecciones cuando el cuadro se asocia con alteraciones cognitivas y de la personalidad, y no solo cuando haya focalización neurológica aguda, cambios que se evidenciaron en este caso ${ }^{22}$.

La etiología del ACV en el paciente joven es muy extensa y se basa según la clasificación TOAST (Trial of ORG 10172 IN Acute Stroke Treatment) ${ }^{23}$. Teniendo en cuenta esta clasificación, la edad del paciente (que se encuentra en el rango de mayor incidencia de ACV en adultos jóvenes) y la distribución de los subgrupos etiológicos de los pacientes jóvenes con ACV isquémico, las principales etiologías son (en orden de frecuencia): la disección vascular y la enfermedad de pequeños vasos (13\%), causas aterotrombótica y cardioembólica (9\%), y finalmente otras causas como las de origen indeterminado incompleto (con foramen oval permeable, $8 \%$ ), sin incluir estadísticas importantes a cerca de la relación con procesos infecciosos'. Dentro del enfoque etiológico dirigido al caso clínico teniendo en cuenta las causas más frecuentes, que engloben no solo el ACV sino las alteraciones neurocognitivas, una primera crisis convulsiva y las lesiones características en piel identificadas al examen físico, obligó a descartar la presencia de un cuadro infeccioso, ya que por el tiempo de evolución y resultados de paraclínicos otras causas como la hipoglucemia y los trastornos hidroelectrolíticos estaría descartadas.

La sífilis meningovascular se produce entre $0,3 \%$ al $2,4 \%$ de los pacientes con sífilis latente, con un período de manifestación hasta de 7 años, aunque en personas inmunosuprimidas este periodo puede acortarse incluso a meses posterior al contacto con el Treponema pallidum, el cual tiene una alta afinidad por el LCR con entrada por la circulación cerebral anterior, como en el caso de este paciente ${ }^{10,24}$, siendo 
a su vez más frecuente la arteritis de Heubner, en la que predomina el compromiso de arterias de mediano y gran calibre, caracterizada por proliferación fibroblástica de la íntima, fibrosis de la adventicia y cambios inflamatorios ${ }^{25}$.

Esta es la forma clínica más frecuente de compromiso del sistema nervioso central asociado a sífilis y en más del $50 \%$ de los casos comienza con un período prodrómico de semanas o meses de duración, que consiste en cefalea, vértigo, cambios de personalidad, insomnio o crisis convulsivas, síntomas similares a los que presentó el paciente ${ }^{26}$. Luego aparece el cuadro vascular cuya clínica es superponible con otros cuadros cerebrovasculares, siendo producto de un fenómeno obstructivo hasta en el $43 \%$ y por arteritis en el $29 \%$, con una edad de presentación que suele ser antes de los 50 años ${ }^{17}$.

Así pues, para el diagnóstico de la neurosífilis se deben tener en cuenta tres componentes importantes: un cuadro clínico concordante con neurosífilis, presentar reactividad de las pruebas serológicas séricas (VDRL/RPR y FTA-ABS) y tener una prueba no treponémica positiva en el LCR; criterios que hicieron parte del diagnóstico del paciente ${ }^{27}$.

Al diagnosticar este tipo de enfermedades de trasmisión sexual, el clínico tiene la obligación de realizar una búsqueda activa de otras entidades de este tipo tales como hepatitis B y C, y VIH, siendo este último confirmado en el paciente. Esta situación puede dificultar el diagnóstico de neurosífilis dada la similitud en las características del LCR, siendo estas inherentes a la propia enfermedad o al uso de la terapia antirretroviral. No existe claridad sobre los valores de proteinorraquia indicado en cada entidad; sin embargo, se ha sido permisivo con los valores indicando una normalidad en las proteínas cuando se cursa con VIH de entre 10 a 20 células por microlitro ${ }^{19}$. Actualmente el método más utilizado es la prueba no treponémica del VDRL en LCR (realizada en el paciente) presentando una alta especificidad (95$100 \%$ ) pero con una baja sensibilidad (varía entre $40 \%$ y $91 \%$ ), debido a los falsos negativos secundarios al efecto prozona ${ }^{19}$. Por lo anterior, es indispensable aumentar la probabilidad diagnóstica teniendo una adecuada historia clínica, un detallado examen físico en búsqueda de los hallazgos típicos de la enfermedad; ya que la prueba inmunológica de oro para el diagnóstico de la neurosífilis es la PCR para síflis en LCR, prueba no disponible en Colombia ${ }^{28}$.
Actualmente el tratamiento recomendado para la neurosífilis sigue siendo la penicilina cristalina a dosis de 3 y 4 millones de unidades cada 4 horas o infusión continua endovenosa de 24 millones de unidades, entre 14 y 21 días, suministrando esquema corto en el paciente dada la respuesta clínica y serológica observada en el LCR de control ${ }^{28}$. Se recomienda el esquema a base de penicilina cristalina inclusive en pacientes con alergia a la penicilina, para quienes se debe realizar desensibilización para su posterior tratamiento. Luego del esquema endovenoso previamente indicado se recomienda complementar esquema con penicilina $G$ benzatínica 2'400.000 unidades intramuscular cada semana por 3 dosis ante posibilidad de que algunos treponemas sobrevivan al esquema inicial y por sus características replicativas que le permiten tener largos períodos de latencia y posterior replicación?.

En general, la neurosífilis puede resultar en daño irreversible del sistema nervioso central. En consecuencia, el objetivo del tratamiento es detener la progresión de la enfermedad; sin embargo, una vez establecido el déficit, la mejoría neurológica con el tratamiento es parcial, como en este caso; lo que hace de vital importancia clínica la necesidad del diagnóstico precoz e inicio temprano del tratamiento para prevenir las secuelas neurológicas.

\section{Conclusión}

Para el enfoque etiológico del paciente joven con síntomas y signos neuropsiquiátricos compatibles con ACV resaltamos la importancia de la realización de un interrogatorio dirigido, teniendo en cuenta aspectos epidemiológicos relevantes como el grupo etario, lugar de procedencia, conductas de riesgo, entre otros, así como un examen físico exhaustivo, en donde para este caso las manifestaciones cutáneas principalmente a nivel de palmas y plantas fueron cardinales para el direccionamiento en la secuencia diagnóstica de la presencia de sífilis, una enfermedad que dadas las características variables en su presentación es conocida en la literatura como la gran simuladora, pero que cuenta con pruebas diagnósticas ampliamente disponibles y económicas que permiten racionalizar los recursos y dar un tratamiento oportuno a este proceso infeccioso con el propósito de disminuir las posibles complicaciones agudas y crónicas en todas sus dimensiones. 


\section{Consideraciones éticas}

El presente caso se publica con consentimiento firmado del paciente.

\section{Conflicto de interés}

Los autores declaran no tener ningún tipo de conflicto de interés.

\section{Financiación}

Se declara que no hay ningún tipo de financiación para la realización del presente caso.

\section{Referencias bibliográficas}

1. Ekker MS, Boot EM, Singhal AB, et al. Epidemiology, aetiology, and management of ischaemic stroke in young adults. Lancet Neurol. 2018;17(9):790-801. doi:10.1016/S1474-4422(18)30233-3

2. Stack CA, Cole JW. A Diagnostic Approach to Stroke in Young Adults. Curr Treat Options Cardiovasc Med. 2017;19(11):84. doi:10.1007/s11936-017-0587-6

3. Putaala J. Ischemic stroke in the young: Current perspectives on incidence, risk factors, and cardiovascular prognosis. Eur Stroke J. 2016;1(1):28-40. doi:10.1177/2396987316629860

4. Romero L, Cigarruista Y, Mackay P, Sánchez R, Serrano A, Vega I et al. Factores asociados a enfermedad cerebrovascular en adultos. Rev Médico Científica. 2013;2(26):39-48. www. revistamedicocientifica.org. Accessed July 24, 2019.

5. Observatorio Nacional de Salud. Carga de enfermedad por enfermedades crónicas no transmisibles y discapacidad en Colombia. Informe técnico. Ministerio de Salud. Biblioteca Digital. doi:ISSN: 2346-3325

6. Chraa M, Louhab N, Kissani N. Stroke in young adults: about 128 cases. Pan Afr Med J. 2014;17:37. doi:10.11604/ pamj.2014.17.37.3226

7. Peeling RW, Mabey D, Kamb ML, Chen X-S, Radolf JD, Benzaken AS. Syphilis. Nat Rev Dis Prim. 2017;3(1):17073. doi:10.1038/ nrdp.2017.73

8. Newman L, Rowley J, Vander Hoorn S, et al. Global Estimates of the Prevalence and Incidence of Four Curable Sexually Transmitted Infections in 2012 Based on Systematic Review and Global Reporting. Meng Z, ed. PLoS One. 2015;10(12):e0143304. doi:10.1371/journal.pone.0143304

9. Singh AE, Romanowski B. Syphilis: review with emphasis on clinical, epidemiologic, and some biologic features. Clin Microbiol Rev. 1999;12(2):187-209. http://www.ncbi.nlm.nih.gov/ pubmed/10194456. Accessed July 24, 2019.

10. Chen M-H, Lin C-C, Lin T-Y, Sung Y-F. Neuro-syphilis, HIV infection and brainstem infarction. QJM. 2013;106(11):10391040. doi:10.1093/qjmed/hct125

11. Golden MR, Marra CM, Holmes KK. Update on Syphilis. JAMA. 2003;290(11):1510. doi:10.1001/jama.290.11.1510
12. Perez Barragán E, Urdez Hernández E, Pérez Orozco B, Sánchez González M. Meningovascular neurosyphilis with basilar artery thrombosis in HIV patient. J Infect Public Health. 2018;11(3):439441. doi:10.1016/J.JIPH.2017.09.009

13. Patira R, Smith-Benjamin S, Wang JJ. Stroke in a young patient with neurosyphilis and HIV. Int J STD AIDS. 2017;28(3):306-309. doi:10.1177/0956462416665029

14. Wolters EC. Neurosyphilis: A Changing Diagnostic Problem? Eur Neurol. 1987;26(1):23-28. doi:10.1159/000116306

15. Pezzini A, Gulletta M, Pinelli L, et al. Meningovascular Syphilis: A Vascular Syndrome with Typical Features? Cerebrovasc Dis. 2001;11(4):352-353. doi:10.1159/000047667

16. Mitsonis C, Kararizou E, Dimopoulos $\mathrm{N}$, et al. Incidence and Clinical Presentation of Neurosyphilis: A Retrospective Study of 81 Cases. Int J Neurosci. 2008;118(9):1251-1257. doi:10.1080/00207450701239426

17. Conde Sendín MÁ, Hernández Fleta JL, Cárdenes Santana MÁ, Amela Peris R. Neurosífilis: formas de presentación y manejo clínico. Rev Neurol. 2002;35(04):380. doi:10.33588/ rn.3504.2001422

18. Nordenbo AM, Sørensen PS. The incidence and clinical presentation of neurosyphilis in Greater Copenhagen 1974 through 1978. Acta Neurol Scand. 2009;63(4):237-246. doi:10.1111/j.1600-0404.1981.tb00777.x

19. Guerrero-Vega J, Ariza-Serrano LM, Ortiz P. Enfoque práctico de la neurosífilis meningovascular: a propósito de un caso presuntivo. Acta Neurológica Colomb. 2016;32(3):233-237. http://www.scielo.org.co/scielo.php?script=sci_arttext\&pid $=$ S0120-87482016000300010. Accessed July 27, 2019 .

20. Uribe CS, Muñoz AI, Arena A IR. Mielitis sifílica aguda. Acta Med Col. http://www.actamedicacolombiana.com/anexo/ articulos/02-1987-06.htm. Published 1987. Accessed July 27, 2019.

21. Andrés J, Ladino LD, Uribe CS, et al. Neurosífilis meningovascular con trombosis de la arteria basilar. Biomédica Rev del Inst Nac Salud. 2012;32(1):8-12. http://www.scielo.org.co/scielo. php?script $=$ sci_arttext\&pid $=$ S0120-41572012000100002. Accessed July 27, 2019.

22. Smajlovic D. Strokes in young adults: epidemiology and prevention. Vasc Health Risk Manag. 2015;11:157. doi:10.2147/ VHRM.S53203

23. Adams HP, Bendixen BH, Kappelle; L Jaap, et al. Classification of Subtype of Acute Ischemic Stroke Definitions for Use in a Multicenter Clinical Trial. http://ahajournals.org. Accessed July 27, 2019.

24. Simms I, Fenton KA, Ashton M, et al. The re-emergence of syphilis in the United Kingdom: the new epidemic phases. Sex Transm Dis. 2005;32(4):220-226. doi:10.1097/01.olq.0000149848.03733. c1

25. Liu L-L, Zheng W-H, Tong M-L, et al. Ischemic stroke as a primary symptom of neurosyphilis among HIV-negative emergency patients. J Neurol Sci. 2012;317(1-2):35-39. doi:10.1016/j. jns.2012.03.003

26. Timmermans M, Carr J. Neurosyphilis in the modern era. J Neurol Neurosurg Psychiatry. 2004;75(12):1727-1730. doi:10.1136/ jnnp.2004.031922

27. Gerstoft J, Lea Katzenstein T, Skov Jensen J. Syphilis and HIV CoInfection. Copenhagen; 2015. http://ugeskriftet.dk/files/scientific_ article_files/2018-11/b5176.pdf. Accessed July 27, 2019.

28. Workowski K BG. Sexually Transmitted Diseases Treatment Guidelines. Centers for Disease Control and Prevention. https:/ www.cdc.gov/mmwr/preview/mmwrhtml/rr6403a1.htm. Published 2015. Accessed July 27, 2019. 